\title{
Correction to: Ocular Adnexal Lesions: A Clinical, Radiological and Pathological Correlation
}

Shantha Amrith, Gangadhara Sundar, and Stephanie Ming Young

\section{Correction to:}

S. Amrith et al. (eds.), Ocular Adnexal Lesions, https://doi.org/10.1007/978-981-13-3798-7

The book was inadvertently published with error and the same has been updated later. A reference has been added "Rootman J, Stewart B, Goldberg RA. Orbital surgery: a conceptual approach. 2nd ed. Philadelphia: Wolters Kluwer Lippincott Williams \& Wilkins; 2014." in the preface in front matter. 